\title{
Avifauna associada a ambientes de influência marítima no litoral de Santa Catarina, Brasil
}

\author{
Joaquim Olinto Branco, Irece Farina Machado \& Marcos Siqueira Bovendorp
}

Centro de Ciências Tecnológicas da Terra e do Mar, Universidade do Vale do. Caixa Postal 360, 88301-970 Itajaí, Santa

Catarina, Brasil. E-mail: branco@cttmar.univali.br

\begin{abstract}
Avifauna associated to environments subjected to marine influence along Santa Catarina Coast, Brazil. Informations about birds associated to environments subjected to marine influence are scarce in Santa Catarina State, despite its $561.4 \mathrm{~km}$ and approximately 300 beaches and several estuaries. Trimestral bird censuses were realized from April/2002 to May/2003 with the purpose of determining the composition and abundance of bird groupings, diversity, equitability and similarity along Santa Catarina coast. A total of 28 censuses were realized, four in each site. It was registered the occurrence of 62 species in mixed groups, where the coastal seabirds and some limnological birds were the most frequent and abundant ones.
\end{abstract}

KEY WORDS. Limnological birds, mixed groups, seabirds.

RESUMO. Apesar dos $561,4 \mathrm{~km}$ e de aproximadamente 300 praias e vários estuários ao longo da costa catarinense, informações sobre as aves associadas aos ambientes de influência marítima são escassos. Durante o período de abril/2002 a maio/2003 foram realizados censos trimestrais na avifauna com o objetivo de determinar a composição e abundância dos agrupamentos, diversidade, equitabilidade e similaridade ao longo do litoral de Santa Catarina. Foram realizados 28 censos, sendo efetuados quatro por sítio e registradas a ocorrência de 62 espécies em bandos mistos, onde as aves marinhas costeiras e algumas limícolas foram as mais freqüentes e abundantes.

PALAVRAS CHAVE. Aves costeiras, aves limícolas, bandos mistos.

Apesar dos 561,4 km de litoral e de aproximadamente 300 praias e vários estuários ao longo da costa catarinense, informações sobre as aves associadas aos ambientes de influência marítima estão restritos, principalmente às ilhas costeiras (Bege \& PAuli 1988, SoAres \& SChiefler 1995a, b, Branco 2003a, b), às praias de Navegantes e Laguna (Schiefler \& SoAres 1994) e ao estuário do Saco da Fazenda (Itajaí) (Branco 2000, 2002, BRAnCo \& EBERT 2002).

Praias arenosas e estuários atuam como locais de alimentação e repouso para bandos de aves aquáticas, onde muitas espécies migratórias armazenam energias necessárias à migração nesses ambientes (VoORen \& ChIARADia 1990).

Este trabalho tem como objetivo caracterizar as comunidades de aves associadas aos ambientes de influência marítima no litoral de Santa Catarina, fornecendo informações sobre abundância, diversidade e finalidade dos agrupamentos dessas aves.

\section{MATERIAL E MÉTODOS}

O litoral de Santa Catarina estende-se da Ilha Saí-guaçú

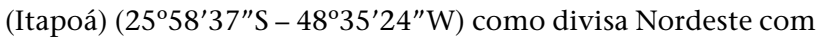
o Estado do Paraná, seguindo até a Foz do Rio Mampituba (Passos de Torres) (29 $\left.18^{\prime} 18^{\prime \prime} \mathrm{S}-49^{\circ} 42^{\prime} 02^{\prime \prime} \mathrm{W}\right)$ como limite Sudeste com o Rio Grande do Sul (Santa Catarina 1991).
Na escolha dos locais de amostragens foram considerados a diversidade e abundância dos bandos de aves, a localização geográfica e a possibilidade de acesso ao longo do ano, sendo selecionados sete localidades: (Araranguá) Foz do Rio Araranguá e praia $\left(28^{\circ} 54^{\prime} 15^{\prime \prime} \mathrm{S}-48^{\circ} 19^{\prime} 03^{\prime \prime} \mathrm{W}\right)$; (Baía Sul) Foz

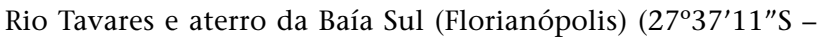
$48^{\circ} 31^{\prime} 46^{\prime \prime}$ ); (São José) Foz do Rio Sergey e Praia de Furnas $\left(27^{\circ} 36^{\prime} 15^{\prime \prime} \mathrm{S}-48^{\circ} 35^{\prime} 51^{\prime \prime} \mathrm{W}\right)$; (Tijucas) Foz do Rio Tijucas e praia

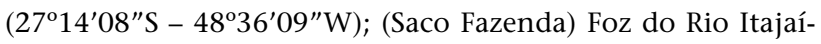
açú e Estuário Saco da Fazenda (Itajaí) (2654'39"S 48 39'04"W); (Barra Velha) Foz do Rio Itapocú e praia (26 34'03"S - 48 39'07"W); e (Barra do Sul) Canal do Lingua-

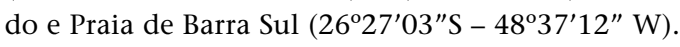

Devido ao número de áreas e a distância entre os extremos dos sítios, optou-se por visitas trimestrais, durante o período de abril/2002 a maio/2003, sendo realizados 28 censos, efetuados quatro por área de estudo. A avifauna associada aos ecossistemas de influência marítima (estuários, praias e dunas) considerada nesse estudo, restringiu-se às espécies que utilizam os locais de amostragens para repouso e/ou alimentação, não sendo computadas as aves marinhas oceânicas que freqüentemente são encontradas moribundas ou mortas nas praias. 
Em cada área, as contagens das aves avistadas tiveram uma duração média de três horas, mantendo-se o mesmo horário de início. A identificação das espécies foi através da observação direta com binóculos (10×50) e nos casos de dúvida, foram fotografadas e comparadas com osmanuais específicos. Foram registrados o número de exemplares por espécie e a finalidade dos agrupamentos (repouso/alimentação).

De acordo com a ocorrência nos censos, independente do número de exemplares, as espécies foram agrupadas em três categorias: constante (c) presente nas quatro estações do ano; ocasional (o) avistadas em três estações; acidental (a) com ocorrência em uma a duas estações; bem como, informada a possível origem decada espécie: visitante meridional (VM); visitante setentrional (VS); residente (RE), espécies com reprodução conhecida no Estado; de situação incerta (IN), sem referência de reprodução confirmada para o litoral catarinense (M oraes \& KRUL 1995). A nomenclatura científica adotada para designar as espécies foi de acordo com Sick (1997).

A similaridade da avifauna entre os locais estudados foi estimada através do índice de Jaccard (Southwood 1968) sendo o número de exemplares por espécie, local e estação do ano utilizados para cal cular os índices de diversidade de Shannon (H') e equitabilidade de Pielou (J') (Ludwig \& ReYnolds 1988).

Em novembro/2002 na Praia de Araranguá, foram observados 10 casais deSterna superciliaris Vieillot, 1819, em processo de reprodução, sendo registrada biometria dos ovos efilhotes e registrados aspectos do ninho.

\section{RESULTADOS}

\section{Composição e sazonalidade}

Foram registradas a ocorrência de 62 espécies, pertencentes à 52 gêneros e $\mathbf{2 8}$ famílias de aves no litoral de Santa Catarina (Tab. I).

Em Araranguá foi registrada a presença de 29 espécies, sendo que os trinta-réis Sterna eurygnatha Saunders, 1876 e S. hirundinacea Lesson, 1831 foram as espécies mais abundantes; na Baía Sul foram observadas 24 espécies, com domínio de Rynchops niger Linnaeus, 1758; em São José constatou-se um incremento para 31 espécies, com destaque para Charadrius semipalmatus Bonaparte, 1825 e a manuten ção deS eurygnatha na segunda posição. Em Tijucas e Saco da Fazenda ocorreu o maior número de espécies (43), com domínio da gaivotaLarus dominicanus Lichtenstein, 1823 e do biguá Phalacrocorax brasilianus (Gmelin, 1789); em Barra Velha foram observadas 29 espécies, com predomínio de L. dominicanus eS. eurygnatha; eem Barra Sul foi registrada a ocorrência de 27 espécies, sendo L. dominicanus eR. niger as mais abundantes (Tab. I).

A ocorrência das espécies constantes oscilou entre os locais de censo, com as menores freqüências em Barra Velha $(6,90 \%)$ e as maiores no Saco da Fazenda (37,20\%); enquanto que nas espécies ocasionais, as menores freqüências ocorreram no Saco da Fazenda $(11,64 \%)$ e as maiores em Araranguá $(27,59 \%)$; já nas acidentais as menores freqüências foram registradasem São José(41,94\%) easmaiores em Tijucas(69,77\%) (Fig. 1, Tab. I).

Das 62 espécies registradas, apenas a gaivotal. dominicanus apresentou ocorrência constante em todos os sítios de amostragens (Tab. I). Destas espécies, 45 foram consideradas residentes, sete de situação incerta, oito visitantes setentrionais e duas visitantes meridionais (Tab. I).

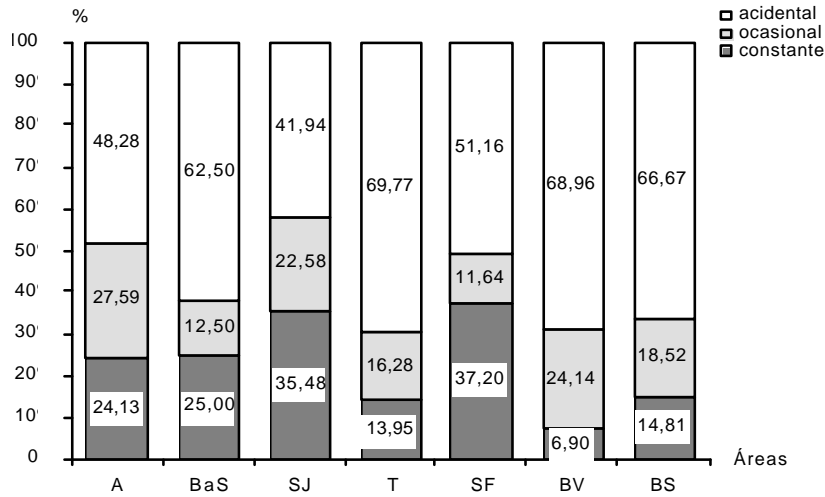

Figura 1. Ocorrência relativa das categorias de espécies nas áreas decenso. (A) Araranguá, (BaS) Baía Sul, (SJ) São José, (T ) Tijucas, (SF) Saco da Fazenda, (BV) Barra Velha, (BS) Barra Sul.

\section{Diversidade e equitabilidade}

Os índices de diversidade ( $\left.\mathrm{H}^{\prime}\right)$ e equitabilidade (J') apre sentaram flutuações moderadas ao longo do ano e variações diferenciadas entre as áreas de censos (Fig. 2). Em geral, os maiores valores de $\mathrm{H}^{\prime}$ e J' foram registrados durante os meses de outono e inverno e os menores entre a primavera e verão, embora durante o verão na Barra Sul, na primavera-verão no Saco da Fazenda e no outono-inverno em Barra Velha, a avifauna não manteve uma un iformidade entre o número de exemplares e de espécies (Fig. 2).

\section{Similaridade entre as áreas}

De acordo com o índice de Jaccard, a similaridade entre as áreas amostradas variou de 36,73 a 75,00\% (Tab. II), sendo que as maiores similaridades da avifauna ocorreram entre Araranguá - Baía Sul (75,00\%), Barra Velha - Barra do Sul $(64,70 \%)$, Araranguá - Barra Vel ha $(61,11 \%)$, enquanto queas menores ficaram entre Baía Sul - Saco da Fazen da (36,73\%) e Araranguá - Saco da Fazenda (38,46\%) (Tab. II).

\section{Agrupamentos multiespecíficos}

Foram registrados a ocorrência de bandos mistos em todas as áreas de estudo e estações do ano, com os mai ores agrupamentos ocorrendo no Saco da Fazenda, durante o outono (13 espécies) e inverno (12 espécies), e os menores em Tijucas, na primavera (duas espécies) e Barra do Sul, no verão (três espécies) (Tab. III). 
Tabela I. Relação das espécies de aves e suas respectivas freqüências nas áreas de censo. A ocorrência (oc) das espécies nos censos é representada por: (c) constante, (o) ocasional, (a) acidental. Origem das espécies: (VM) visitante meridional, (VS) visitante setentrional, (RE) residente, (IN) de situação incerta).

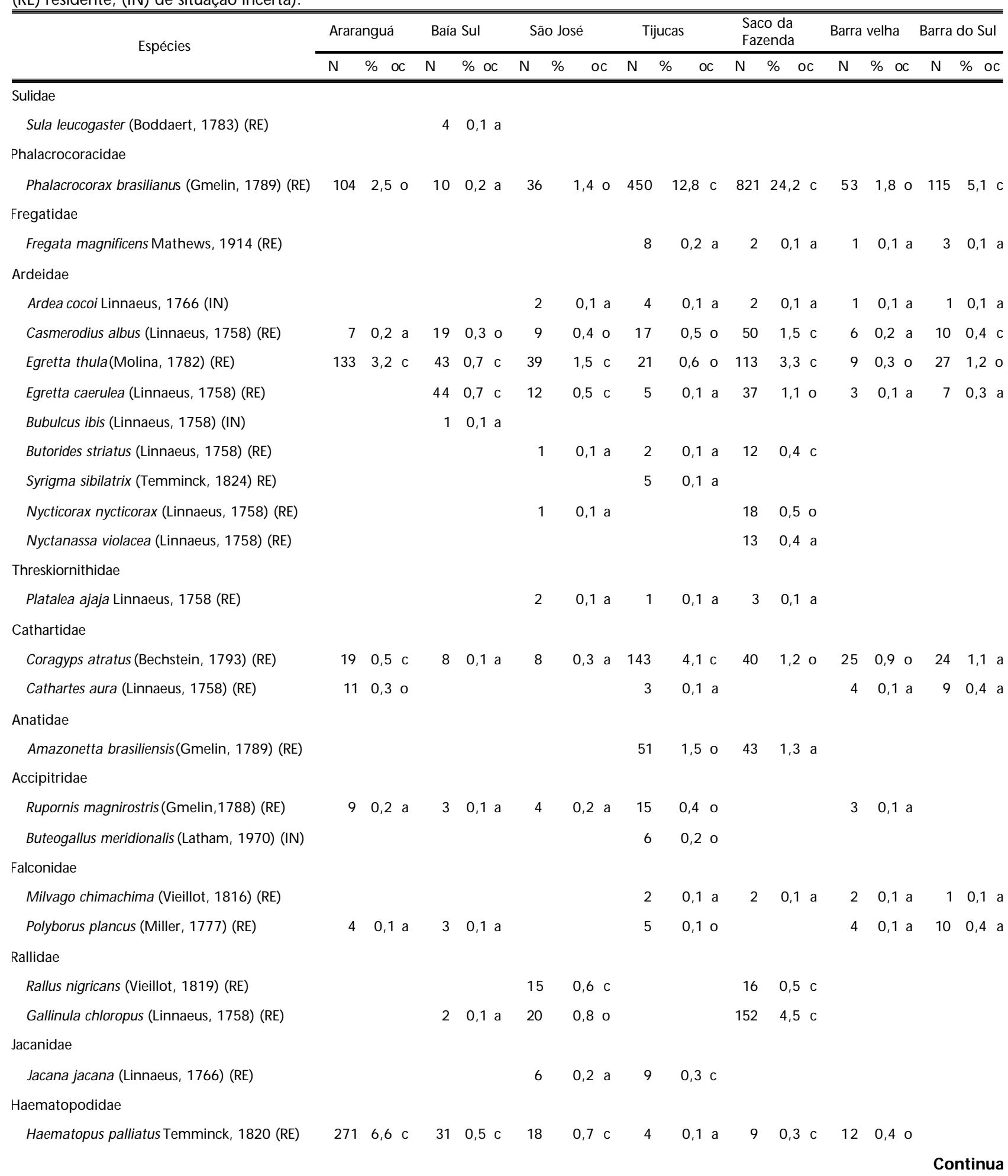


Tabela I. Continuação.

\begin{tabular}{|c|c|c|c|c|c|c|c|c|c|c|c|c|c|c|c|c|c|}
\hline \multirow{2}{*}{ Espécies } & \multicolumn{2}{|c|}{ Araranguá } & \multicolumn{3}{|c|}{ Baía Sul } & \multicolumn{2}{|c|}{ São José } & \multicolumn{4}{|c|}{ Tijucas } & \multicolumn{2}{|c|}{$\begin{array}{l}\text { Saco da } \\
\text { Fazenda }\end{array}$} & \multicolumn{4}{|c|}{ Barra velha Barra do Su } \\
\hline & $\mathrm{N}$ & $\%$ oc & $\mathrm{CN}$ & & $\%$ oc & $\mathrm{N}$ & $\%$ & $x \quad 1$ & $\mathrm{~N}$ & $\%$ & OC & $\mathrm{N}$ & $\%$ oc & $\mathrm{N}$ & $\%$ oc & $c \mathrm{~N}$ & $\%$ oC \\
\hline \multicolumn{18}{|l|}{ Charadriidae } \\
\hline Vanellus chilensis (Molina, 1782) (RE) & 25 & 0,6 & c & 69 & $1,1 \mathrm{c}$ & 343 & 13,5 & c & 51 & 1,5 & c & 132 & $3,9 \mathrm{c}$ & 15 & $0,5 \circ$ & $0 \quad 20$ & 0,9 o \\
\hline Pluvialis dominica (Müller, 1776) (VS) & 50 & 1,2 & a & & & 6 & 0,2 & a & 58 & 1,7 & & & & & & & \\
\hline Charadrius semipalmatus Bonaparte, 1825 (VS) & 32 & 0,77 & $0 \quad 10$ & 081, & ,68 a & 670 & 26,43 & c 3 & 389 & 11,09 & o & 44 & $1,30 \circ$ & 9 & 0,310 & o 20 & 0,89 \\
\hline Charadrius collaris Vieillot, 1818 (RE) & 19 & 0,50 & & & & & & & 2 & 0,1 & a & & & 3 & $0,1 \mathrm{a}$ & & \\
\hline \multicolumn{18}{|l|}{ Scolopacidae } \\
\hline Tringa flavipes (Gmelin, 1789) (VS) & 24 & 0,6 & & & & 2 & 0,1 & a & 1 & 0,1 & a & & & & & & \\
\hline Actitis macularia (Linnaeus, 1766) (VS) & 2 & 0,1 & & & & 96 & 3,8 & 0 & 61 & 1,7 & & 5 & $0,1 \mathrm{a}$ & & & & \\
\hline Calidris canutus (Linnaeus, 1758) (VS) & 80 & 1,9 & & & & 46 & 1,8 & a & & & & 8 & 0,2 a & a & & 2 & 0,1 \\
\hline Calidris alba (Pallas, 1764) (VS) & 5 & 0,1 & & & & & & & & & & & & & & & \\
\hline Numenius phaeopus (Linnaeus, 1758) (VS) & & & & & & & & & 7 & 0,2 & & & & & & & \\
\hline \multicolumn{18}{|l|}{ Recunvirostridae } \\
\hline Himantopus himantopus (Linnaeus, 1758) (IN) & 209 & $5,1 \mathrm{c}$ & & 62 & 2,5 a & 135 & 5,3 & 03 & 304 & 8,7 & & 11 & 0,3 a & a & & & \\
\hline \multicolumn{18}{|l|}{ Laridae } \\
\hline Larus dominicanus Lichtenstein, 1823 (RE) & 254 & 6,2 & c 16 & 62 & $2,5 \mathrm{c}$ & 90 & 3,5 & c 12 & 1255 & 35,8 & c & 733 & $21,6 \mathrm{c}$ & C 1560 & $53,0 \mathrm{c}$ & c 844 & 37,4 \\
\hline Larus maculipennisLichtenstein, 1823 (VM) & 8 & 0,2 & & & & & & & 94 & 2,7 & & 6 & $0,2 \mathrm{a}$ & a & & & \\
\hline Sterna hirundinacea Lesson, 1831 (RE) & 392 & 9,60 & & 16 & 0,3 a & & & & & & & 4 & $0,1 \mathrm{a}$ & a 163 & 5,5 a & a 365 & 16,2 \\
\hline Sterna hirundo Linnaeus, 1758 (VS) & 255 & 6,2 & & & & & & & 4 & 0,1 & a & & & 35 & 1,2 a & & \\
\hline Sterna trudeaui Audubon, 1938 (VM) & 39 & 0,90 & & 3 & 0,1 a & 104 & 4,1 & o & 5 & 0,1 & a & 71 & $2,1 \mathrm{a}$ & 25 & 0,8 a & a 21 & $1 \quad 0,9$ a \\
\hline Sterna superciliaris Vieillot, 1819 (RE) & 57 & 1,4 & & & & & & & & & & & & 2 & $0,1 \mathrm{a}$ & & \\
\hline Sterna maxima Boddaert, 1783 (IN) & 14 & 0,3 & 0 & 15 & 0,2 a & 1 & 0,1 & a & 5 & 0,1 & a & 11 & 0,3 a & 66 & $2,3 \circ$ & $0 \quad 15$ & $0,7 \circ$ \\
\hline Sterna eurygnatha Saunders, 1876 (RE) & 1987 & 48,6 & c 38 & 866 & $6,0 \circ$ & 425 & 16,8 & c & 69 & 2,0 & $a$ & 236 & 6,9 a & a 892 & $30,3 \mathrm{c}$ & c 316 & $14,1 \mathrm{c}$ \\
\hline \multicolumn{18}{|l|}{ Rynchopidae } \\
\hline Rynchops niger Linnaeus, 1758 (IN) & 61 & 1,5 & o 530 & 0582 & $2,5 \mathrm{c}$ & 257 & 10,2 & c 3 & 346 & 9,9 & $a$ & 310 & 9,10 & 8 & $0,3 a$ & a 376 & 16,7 \\
\hline \multicolumn{18}{|l|}{ Columbidae } \\
\hline Columbina talpacoti (Temminck, 1811) (RE) & & & & & & & & & 9 & 0,3 & & 51 & $1,5 \mathrm{c}$ & & & & \\
\hline Columbina picui (Temminck, 1813) (RE) & & & & & & & & & & & & 5 & 0,2 a & & & & \\
\hline \multicolumn{18}{|l|}{ Cuculidae } \\
\hline Crotophaga ani Linnaeus, 1758 (RE) & & & & & & & & & 12 & 0,3 & a & 6 & $0,2 \mathrm{a}$ & a & & & \\
\hline Guira guira (Gmelin, 1788) (RE) & & & & 12 & 0,2 a & & & & 16 & 0,5 & & & & & & 8 & $3 \quad 0,4$ a \\
\hline \multicolumn{18}{|l|}{ Strigidae } \\
\hline Speotyto cunicularia (Molina, 1782) (RE) & & & & 9 & $0,1 \circ$ & & & & 27 & 0,8 & & & & 13 & $0,4 \quad a$ & & \\
\hline \multicolumn{18}{|l|}{ Alcedinidae } \\
\hline Ceryle torquata (Linnaeus, 1766) (RE) & 2 & 0,1 & & 1 & 0,1 a & & & & 2 & 0,1 & a & 4 & $0,1 \mathrm{a}$ & 4 & $0,1 \mathrm{a}$ & 7 & 0,3 \\
\hline Chloroceryle americana (Gmelin, 1788) (IN) & & & & & & & & & & & & & & 1 & L $\quad 0,1$ a & & \\
\hline \multicolumn{18}{|l|}{ Furnariidae } \\
\hline Furnarius rufus (Gmelin,1788) (RE) & 8 & 0,2 & & & & 32 & 1,3 & c & 4 & 0,1 & a & 43 & $1,3 \mathrm{c}$ & c 12 & 0,4 a & a 12 & 0,5 a \\
\hline
\end{tabular}

Revista Brasileira de Zoologia 21(3): 459-466, setembro 2004 
Tabela I. Continuação.

\begin{tabular}{|c|c|c|c|c|c|c|c|c|c|c|c|c|c|c|c|c|c|}
\hline \multirow{2}{*}{ Espécies } & \multicolumn{2}{|c|}{ Araranguá } & \multicolumn{2}{|c|}{ Baía Sul } & \multicolumn{3}{|c|}{ São José } & \multicolumn{3}{|c|}{ Tijucas } & \multicolumn{3}{|c|}{$\begin{array}{l}\text { Saco da } \\
\text { Fazenda }\end{array}$} & \multicolumn{4}{|c|}{ Barra velha Barra do Su } \\
\hline & $\mathrm{N}$ & $\%$ oc & $\mathrm{N}$ & $\%$ oc & $\mathrm{N}$ & $\%$ & OC & $\mathrm{N}$ & $\%$ & OC & $\mathrm{N}$ & $\%$ & OC & $\mathrm{N}$ & $\%$ oc & $\mathrm{N}$ & $\%$ oc \\
\hline \multicolumn{18}{|l|}{ Tyrannidae } \\
\hline Machetornis rixosus (Vieillot, 1819) (RE) & & & & & & & & & & & 18 & 0,5 & $5 \mathrm{c}$ & & & 2 & 0,1 a \\
\hline Pitangus sulphuratus (Linnaeus, 1766) (RE) & 10 & 0,2 a & 19 & $0,3 a$ & 63 & & $2,5 \mathrm{c}$ & 12 & & 0,3 a & 80 & 2,4 & $4 \mathrm{c}$ & 13 & 0,4 a & 20 & 0,9 o \\
\hline Tyrannus melancholicus Vieillot, 1819 (RE) & & & & & & & & & & & 16 & 0,5 & 5 a & & & 4 & 0,2 a \\
\hline \multicolumn{18}{|l|}{ Troglodytidae } \\
\hline Troglodytes aedon Vieillot, 1807 (RE) & & & & & 30 & & $1,2 \circ$ & 6 & & 0,2 a & 39 & 1,2 & $2 c$ & 2 & 0,1 a & 13 & 0,6 a \\
\hline \multicolumn{18}{|l|}{ Muscicapidae } \\
\hline Turdus leucomelas Vieillot, 1818 (RE) & & & & & & & & & & & 16 & 0,5 & 5 a & & & & \\
\hline \multicolumn{18}{|l|}{ Emberizidae } \\
\hline Thraupis sayaca (Linnaeus, 1766) (RE) & & & & & & & & & & & 17 & 0,5 & 5 a & & & & \\
\hline Zonotrichia capensis (Müller, 1776) (RE) & & & & & & & & & & & & & & & & 6 & 0,3 a \\
\hline Sicalis flaveola (Linnaeus, 1766) (RE) & & & & & 25 & & 1,0 a & 3 & 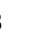 & $0,1 \mathrm{a}$ & 36 & 1,1 & $1 \mathrm{c}$ & & & & \\
\hline Molothrus bonariensis (Gmelin,1789) (RE) & & & & & & & & 16 & & 0,5 a & 3 & 0,1 & 1 a & & & & \\
\hline \multicolumn{18}{|l|}{ Passeridae } \\
\hline Passer domesticus (Linnaeus, 1758)(RE) & & & & & 38 & & 1,5 a & & & & 160 & 4,7 & $7 \mathrm{c}$ & & & & \\
\hline \multicolumn{18}{|l|}{ Estrildidae } \\
\hline Estrilda astrild (Linnaeus, 1758) (RE) & & & & & & & & & & & 6 & 0,2 & $2 a$ & & & & \\
\hline
\end{tabular}

Tabela II. Similaridade da avifauna entre as áreas de censo.

\begin{tabular}{ccccccc}
\hline \hline & Baía Sul & São José & Tijucas & Saco da Fazenda & Barra Velha & Barra Sul \\
\hline \hline \multirow{2}{*}{ Araranguá } & 75,00 & 50,00 & 53,19 & 38,46 & 61,11 & 47,37 \\
& Baía Sul & 44,47 & 42,55 & 36,73 & 55,88 & 50,00 \\
& & $54 a$ José & 54,17 & 57,45 & 42,86 & 41,46 \\
& & & 53,57 & 56,52 & 45,83 \\
& & & & Saco da Fazenda & 41,18 & 48,94 \\
& & & & Barra Velha & 64,70 \\
\hline
\end{tabular}

Tabela III. Número de espécies ( $\mathrm{N}$ s.p) e exemplares ( $\mathrm{N}$ ex.) nos agrupamentos multiespecíficos, da avifauna nas áreas de censo.

\begin{tabular}{|c|c|c|c|c|c|c|c|c|}
\hline \multirow{2}{*}{ Áreas } & \multicolumn{2}{|c|}{ Outono } & \multicolumn{2}{|c|}{ Inverno } & \multicolumn{2}{|c|}{ Primavera } & \multicolumn{2}{|c|}{ Verão } \\
\hline & N sp. & $\mathrm{N}$ ex. & N sp. & $\mathrm{N}$ ex. & N sp. & $\mathrm{N}$ ex. & N sp. & $\mathrm{N}$ ex. \\
\hline Araranguá & 10 & 444 & 12 & 2256 & 9 & 1901 & 5 & 193 \\
\hline Baía Sul & 8 & 2937 & 6 & 247 & 4 & 354 & 7 & 4515 \\
\hline São José & 6 & 489 & 7 & 369 & 7 & 800 & 6 & 590 \\
\hline Tijucas & 8 & 1095 & 6 & 583 & 2 & 221 & 6 & 1385 \\
\hline Saco da Fazenda & 13 & 957 & 12 & 1739 & 9 & 2030 & 10 & 1695 \\
\hline Barra Velha & 6 & 1136 & 9 & 4596 & 5 & 522 & 6 & 415 \\
\hline Barra Sul & 5 & 632 & 5 & 303 & 7 & 1309 & 3 & 578 \\
\hline
\end{tabular}



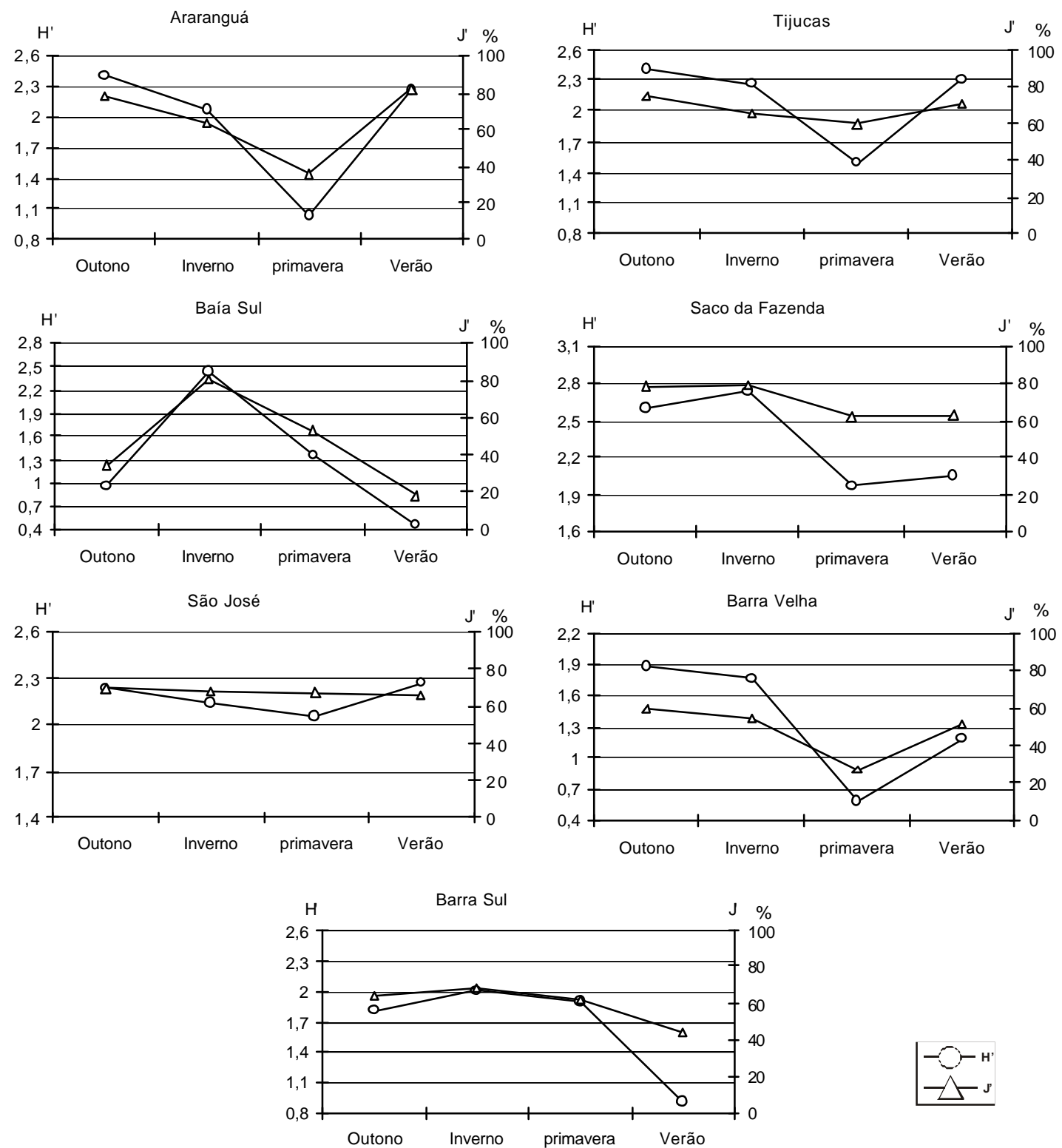

Figura 2. Variação sazonal dos índices de diversidade de Shannon ( $\left.\mathrm{H}^{\prime}\right)$ e equitabilidade de Pielou (J'), da avifauna nas áreas de censo.

Osbandos, em geral, tiveram como finalidadeo repouso ea manutenção da plumagem, entretanto, duranteo inverno em Tijucas e no outono-inverno em São J osé foram intercalados entre períodos de alimentação e de repouso.

As aves marinhas costei ras e al gumas limícolas foram as mais freqüentes e abundantes nos agrupamentos, especial mente L. dominicanus, Sterna eurygnatha, S. trudeaui S. hirundinacea, Rynchops niger, Charadrius semipalmatus, Phalacrocorax brasilianus, Egretta thula (Molina, 1782) eE. caerul ea (Linnaeus, 1758) (Tab. I).

\section{Registro de nidificação}

No censo de 30 de novembro de 2002, na Praia de Araranguá, foi encontrada uma pequena colônia com 10 casais do trinta-réis-anão Sterna superciliaris Vieillot, 1819, nidificando nas dunas próximas da foz do Rio Araranguá. Osninhos foram construídos em pequenas cavidades sobre a areia, onde foram depositados os ovos de coloração marrom-clara com pequenas manchas pretas distribuídas irregularmente, bem camuflados. O comprimento dos ovos variou entre 3,1 a $3,2 \mathrm{~cm}$, com largura de $2,4 \mathrm{~cm}$ e peso de $10 \mathrm{~g}(\mathrm{n}=2)$; enquanto que o compri-

Revista Brasileira de Zoologia 21(3): 459-466, setembro 2004 
mento do cúlmen do bico de dois filhotes, oscilou entre 1,8 a $2,0 \mathrm{~cm}$ e o peso entre 52 a $54 \mathrm{~g}$. Este é o primeiro registro de nidificação deS. superciliaris para o litoral de Santa Catarina.

Nessa ocasião, também foi capturado um ninhego de Haematopus palliatus Temminck, 1829, nas proximidades daárea de nidificação dos trinta-réis. O comprimento do cúlmen do bico desse filhote foi de $3,4 \mathrm{~cm}$ e o peso de $180 \mathrm{~g}$.

\section{DISCUSSÃO}

Em ecossistemas de influência marítima do litoral paranaense, M ORAES \& KRUL (1995) registraram a ocorrência de 59 espécies de aves, enquanto que Schiefler \& Soares (1994), num estudo comparativo sobrea avifauna das praias de Navegantes e Laguna, Santa Catarina, encontraram 32 espécies, sendo 22 comuns às duas praias. Branco (2000) registrou a presença de 45 espécies no estuário do Saco da Fazenda, Santa Catarina) e Vooren \& Chiaradia (1990) na Praia do Cassino, Rio Grande do Sul encontraram 33 espécies. Apesar da maior riqueza aparente (62 espécies) de aves nas praias de Santa Catarina, onde muitas espécies são comuns no litoral da região sul do país, mas quando descontada a participação dos Passeriformes, essa riqueza estaria próxima aos estudos anteriores.

Ao comparar variáveis ecológicas de diversas localidades, constatamos que a maioria dos dados são expressos em val ores totais e osíndices utilizados variam muito; assim como a bibliografia disponível proporcionar, na sua maioria, apenas a informação da riqueza desses sítios através do número de espécies. Entretanto, os valores dos índices de diversidade de Shannon registrados no Saco da Fazenda (Branco 2000) e o de Simpsom nas prai as de Navegantes e Laguna (SCHIEFLER \& SOARES 1994), estão próximos aos obtidos neste estudo. Já o índice desimilaridade de Jaccard, demonstrou uma afinidade de 36,73 a $75,00 \%$ entre as praias do litoral de Santa Catarina, sendo influenciado diretamente pelas flutuações sazonais das populações de aves nessas localidades.

As seis áreas estudadas apresentaram variações sazonais, evidenciando-se um padrão geral de semelhanças, característico entre associações às estações do ano. Esses ti pos de agrupamentos sazonais são comuns em avifauna, tendo sido observado no litoral do Paraná (M ORAES \& KRUL 1995) eno estuário do Saco da Fazenda (Branco 2000).

As aves costei ras pertencentes à Laridae, as limícolas Rynchopidae, Charadriidae, e as aquáticas Ardeidae e Phalacrocoracidae contribuíram com as maiores abundâncias entre as praias amostradas; essa tendência, também foi observada no estuário do Saco da Fazenda (Branco 2000). A dominância de Larus dominicanus em estudos envolvendo aves marinhas costei rasénotória no litoral dos estados do Paraná, Santa Catarina e Rio Grande do Sul (Moraes \& Krul 1995, Schiefler \& Soares 1994, Vooren \& Chiaradia 1990, Branco 2000). Esse predomínio é atribuído a uma dieta generalista e oportunista, sendo capaz de ocupar diferentes nichos e explorar uma gama de presas, bem como fontes de alimentos excedentes das atividades humanas (Giaccardi et al. 1997, Branco 2000, Branco \& Ebert 2002).

De acordo com BARBIERI et al. (2000), o município de llha Comprida, São Paulo, éuma dasáreas de parada para o forrageio e descanso deCharadrius semipalmatus, durante a migração de retorno para o hemisfério norte (abril) e áreas de al imentação no hemisfério sul (setembro). Em Santa Catarina, as maiores abundâncias dessa espécie foram registradas em São José e Tijucas, nos meses de primavera e verão, o que provavelmente realça a ten dência observada no litoral paulista.

Algumas espécies de Passeriformes como: Troglodytes aedon Vieillot, 1807, Turdus leucomelas Vieillot, 1818, Zonotrichia capensis (Müller, 1776), Sicalis flaveola (Linnaeus, 1766), Molothrus bonariensis (Gmelin, 1789), Passer domesticus (Linnaeus, 1758) e Estrilda astrild (Linnaeus, 1758) registradas nos sítios de amostragens, são consideradas aves oportunistas e ocupam vários ambientes, forrageando em áreas com gramíneas das regiões de estuários, praias e dunas.

Em ambiente de influência marítima, agrupamentos multiespecíficos de aves, são formados em decorrência da partilha dealimento, local protegido para o repouso enidificação, assumindo um papel importante na proteção das comunidades (Moraes \& Krul 1995, Branco 2002).

\section{AGRADECIMENTOS}

Aos orientandos Hélio Augusto Alves Fracasso eCristiano Lombardo Evangelista, pelo auxílio nos trabal hos de campo.

\section{REFERÊN CIA S BIBLIOGRÁ FICAS}

Barbierl, E.; J.T. Mendonça \& S.C. Xavier. 2000. Distribuição da batuíra-de-bando (Charadrius semipalmatus) ao longo do ano de 1999 na Praia da Ilha Comprida. NotastécnicasFacimar, Itajaí, 4: 69-76.

Bege, L.A.R. \& B.T.PauLI. 1988. AsavesnasIIhas Moleques do Sul - Santa Catarina: Aspectos da ecologia, etologia e anilhamento de aves marinhas. Florianópolis, FATMA, $64 p$.

Branco, J.O. 2000. Avifauna associada ao estuário do Saco da Fazenda. Revista Brasil ei ra deZool ogia, Curitiba, 17 (2): 387-394.

- 2002. Flutuações sazonais na abundância dePhalacrocorax brasilianus (Gmelin) no estuário do Saco da Fazenda, Itajaí, Santa Catarina, Brasil. Revista Brasil lei ra de Zoologia, Curitiba, 19 (4): 1057-1062.

. 2003a. Reprodução das aves marinhas nas ilhas costeiras de Santa Catarina, Brasil. Revista Brasi leira de Zoologia, Curitiba, 20 (4): 619-623.

ـ 2003b. Reprodução de Sterna hirundinacea Lesson e S. eurygnatha Saunders (Aves, Laridae), no litoral de Santa Catarina, Brasil. Revista Brasil ei ra deZool ogia, Curitiba, 20 (4): 655-659.

Branco, J.O. \& L.A. Ebert. 2002. Estrutura populacional deLarus 
dominicanus Lichtenstein, 1823 no estuário do Saco da Fazenda, Itajaí, Santa Catarina, Brasil. Ararajuba, Rio de Janeiro, 10 (1): 79-82.

LudWIG, J.A. \& J.F. ReYNolds. 1988. Statistical ecology: a primer on methodsand computing. New York, John Wiley \& Sons, $338 p$.

Moraes, V.S. \& R. Krul. 1995. Aves associadas a ecossistemas de influência marítima no litoral do Paraná. Arqui vos de Biologia eTecnologia, Curitiba, 38 (1): 121-134.

Giaccardi, M.; P. Yorio \& E. Lizurume. 1997. Patrones estacionales dela gai vota cocinera (Larus dominicanus) em um basural Patagónico y sus relaciones com el manejo de residuos urbanosy pesqueros. Ornitologia Neotropical , Québec, 8: 77-84.

Santa Catarina. 1991. Atl as escolar de Santa Catarina, Secretaria deEstado de Coordenação Geral e Planejamento, Subsecretaria deEstudos Geográfi cose Estatísticos. Rio deJaneiro, Ed. Aerofoto Cruzeiro, 96p.
Sotres, M. \& A.F. Schiefler. 1995a. Reprodução de Larus dominicanus (Aves, Laridae), na Il hota da Galeta, Laguna, SC, Brasil. Arquivos de Biologia e Tecnologia, Curitiba, 38 (1): 313-316.

Soares, M. \& A.F. Schiefler. 1995b. Aves da Il hota da Galheta, Laguna, SC, Brasil. Arquivos de Biologia e Tecnologia, Curitiba, 38 (4): 1101-1107.

Southwood, T.R.E. 1968. Ecological methods. London, Chapman and Holl, 368p.

Schiefler, A.F. \& M. Soares. 1994. Estudo comparativo da avifauna das praias de Navegantes eLaguna, Santa Catarina. Biotemas, Florianópolis, 7 (1/2): 31-45

Sıck, H. 1997. Ornitologia Brasi leira. Rio de Janeiro, Editora Nova Fronteira, 912p.

Vooren, C. \& A. Chiaradia. 1990. Seasonal abundance and behaviour of costal birds on Cassino Beach, Brazil. Ornitologia Neotropical, Québec, 1: 9-24.

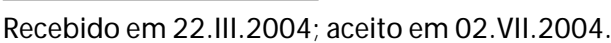

